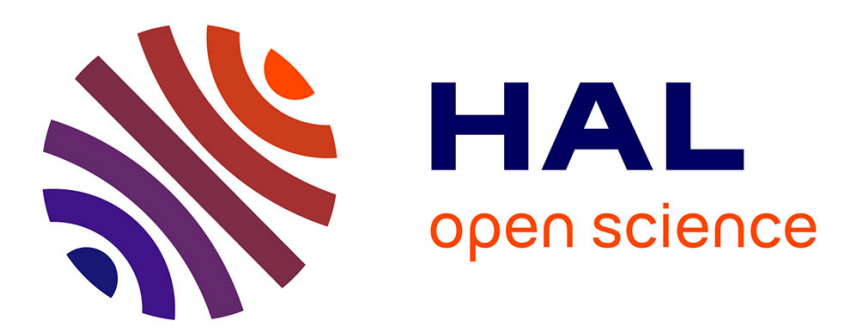

\title{
Semantic enrichment of spatio-temporal trajectories for worker safety on construction sites
}

\author{
Muhammad Arslan, Christophe Cruz, Dominique Ginhac
}

\section{To cite this version:}

Muhammad Arslan, Christophe Cruz, Dominique Ginhac. Semantic enrichment of spatio-temporal trajectories for worker safety on construction sites. Personal and Ubiquitous Computing, 2019, 23 (5-6), pp.749-764. 10.1007/s00779-018-01199-5 . hal-02073566

\section{HAL Id: hal-02073566 https://u-bourgogne.hal.science/hal-02073566}

Submitted on 3 Jun 2019

HAL is a multi-disciplinary open access archive for the deposit and dissemination of scientific research documents, whether they are published or not. The documents may come from teaching and research institutions in France or abroad, or from public or private research centers.
L'archive ouverte pluridisciplinaire HAL, est destinée au dépôt et à la diffusion de documents scientifiques de niveau recherche, publiés ou non, émanant des établissements d'enseignement et de recherche français ou étrangers, des laboratoires publics ou privés. 


\title{
Semantic enrichment of spatio-temporal trajectories for worker safety on construction sites
}

\author{
Muhammad Arslan $^{1}$ - Christophe $\mathrm{Cruz}^{1} \cdot$ Dominique Ginhac $^{2}$
}

\begin{abstract}
Thousands of fatalities are reported from the construction industry every year and a high percentage of them are due to the unsafe worker movements which resulted in falling from heights, transportation accidents, exposure to harmful environments, and striking against or being struck by the moving equipment. To reduce such fatalities, a system is proposed to monitor worker movements on a construction site by collecting their raw spatio-temporal trajectory data and enriching it with the relevant semantic information. To acquire the trajectories, the use of an indoor positioning system (IPS) is considered. Bluetooth beacons are used for collecting spatio-temporal information of the building users. By means of an Android-based mobile application, neighboring beacons' signals are selected, and a geo-localization technique is performed to get the unique pairs of users' location coordinates. After pre-processing this collected data, three semantic enrichment techniques are used to construct semantically enriched trajectories which are as follows: (1) enrichment with the semantic points which maps site location identification to the trajectory points; (2) enrichment with the semantic lines which relies on the speed-based segmentation approach to infer user modes of transportation; (3) enrichment with the semantic region for mapping a complete trajectory on an actual building or a construction site zone. The proposed system will help in extracting multifaceted trajectory characteristics and generates semantic trajectories to enable the desired semantic insights for better understanding of the underlying meaningful worker movements using the contextual data related to the building environment. Generated semantic trajectories will help health and safety (H\&S) managers in making improved decisions for monitoring and controlling site activities by visualizing site-zones' density to avoid congestion, proximity analysis to prevent workers collisions, identifying unauthorized access to hazardous areas, and monitoring movements of workers and machinery to reduce transportation accidents.
\end{abstract}

Keywords Safety $\cdot$ Workers $\cdot$ Construction $\cdot$ Spatio-temporal data $\cdot$ Fatalities $\cdot$ BIM

\section{Introduction}

The construction industry is very hazardous in nature because of the harsh working environment [1] and the involvement of high safety risks [2]. According to the Bureau of Labor Statistics, in 2016, out of 5190 fatal work injuries, $19 \%$ of fatalities were recorded in the US construction industry [3]. The major fatalities were caused by falling from heights [4],

Muhammad Arslan

muhammad.arslan@u-bourgogne.fr

1 Laboratoire d'Informatique de Bourgogne - EA 7534, University Bourgogne Franche-Comté, 9 rue Alain Savary, 21078 Dijon Cedex, France

2 Laboratoire Imagerie et Vision Artificielle - EA 7535, University Bourgogne Franche-Comté, 9 rue Alain Savary, 21078 Dijon Cedex, France transportation accidents [5], exposure to harmful environments [6], and striking against or being struck by the moving equipment [7]. The latest available statistics [3] of fatal occupational injuries by an event or exposure in the construction industry are shown in Fig. 1. A closer look at the latest research reveals that one of the major reasons for construction site accidents is because of the unsafe worker behaviors resulting in serious collisions with site objects and machinery. For instance, limited spatial awareness [7] of the operating machinery within the workers' proximity because of the blind spots and nearby interferences can lead to hazardous situations on sites. To increase the spatial awareness of sites for reducing the accidents, there is a need for more effective construction resources' mobility monitoring and safety planning methods to identify unsafe worker behavioral patterns.

The dynamic interactions of the workers in an uncertain and dynamic construction environment where the building infrastructure is continuously evolving with time often 
Fig. 1 Fatal occupational injuries by an event or exposure in the construction industry (20122016)

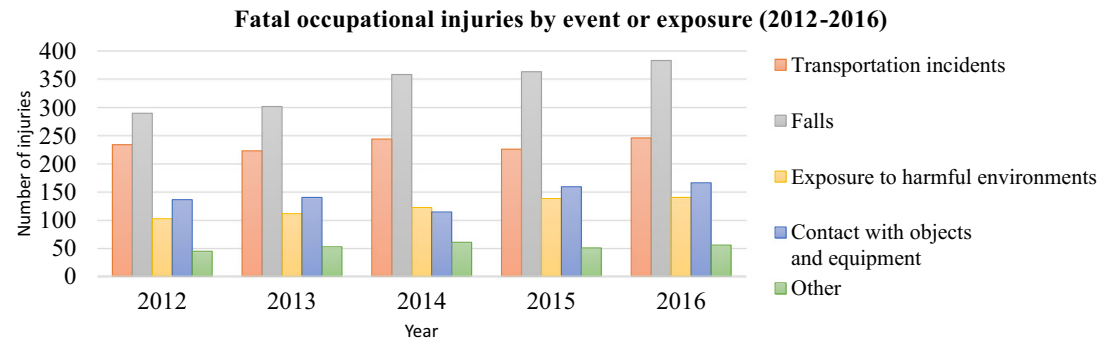

resulted in unsafe worker behaviors [8]. These dynamic interactions raise serious safety concerns for H\&S managers as the movements of construction resources (workers and machinery) are changing according to the change in the building infrastructure $[9,10]$. In the last few decades, to reduce safety hazards, dynamic interactions of the construction resources are tracked in real time using different sensor-based location tracking technologies such as radio-frequency identification (RFID), Global Positioning System (GPS), ultra-wideband (UWB), and vision-based sensing systems [11]. Each one of them has its own benefits and limitations. Among all, the most prominent and widely used tracking technology for construction safety applications is based on the GPS or an IPS $[10,12]$. It is used to record spatio-temporal trajectories as a series of location points as spatio-temporal tuples $\left(x_{i}, y_{i}, t_{i}\right)$ in the form of latitude and longitude coordinates with timestamps generated by a moving object in space [13]. It holds multifaceted attributes, for example, time, a position of an object in the geographical coordinate system, a direction of an object, the speed of an object, a change in direction, acceleration, and distance traveled [14]. These attributes can be extracted directly from spatio-temporal trajectories by applying preprocessing techniques. However, processed spatio-temporal trajectory data cannot provide a clear understanding of the meanings behind workers' mobility behaviors because it lacks semantic information related to the environment [13]. To add semantic information to processed trajectories using external data sources (application databases, geodatabases, etc.) including openly available and private data related to the environment, a semantic enrichment process is required $[13,14]$. The added relevant semantic or contextual information to complement the processed trajectory data using alphanumeric properties is called "annotations." In this paper, three types of annotations are discussed which are as follows: annotating with semantic points (spatio-temporal tuples selected based on the user stay duration), semantic lines (detecting the transportation mode between successive spatio-temporal tuples based on the speed values), and semantic regions (the geographical building or site location where the trajectory takes place). For example, a trajectory of a worker named "John" is going to dump a construction material on a site. The extracted stay locations of "John" are annotated as the semantic points or the point of interests (POIs) where he is spending most of his time. Segmenting each trajectory episode to find the transportation mode used by "John" while traveling between the semantic points is annotated as a semantic line. Moreover, the whole trajectory of "John" will be annotated with a semantic region or region of interest (ROI) where an entire mobility has been taken place to visualize it from the geographical context of a building. In the existing literature, there exist many approaches of semantic enrichment of trajectories. However, these approaches are constructed to understand the outdoor trajectories of moving objects and do not generate semantic trajectories for dynamic indoor environments where the building infrastructure is evolving in its shape, size, and attributes with time. Construction sites are the best example of dynamic environments where the new walls and infrastructure supports are added frequently on sites. Such changes in the building environment are required to be captured by the semantic data model to generate trajectories with the most updated semantic information for studying worker movements in dynamic environments.

For addressing semantic trajectory modeling needs for a dynamic environment using the construction site scenario, our contributions follow (i) trajectories pre-processing subsystem: as trajectories contain multifaceted characteristics including time, position of the object in the geographical coordinate system, direction of the object, speed of the object, change in direction, and distance traveled [13]. A system is developed for extracting such characteristics to better understand the trajectories. Moreover, an identification of the stay points is achieved from workers' trajectories that will help in recognizing important regions in the building. (ii) Semantic enrichment subsystem: for enriching the trajectories with annotations using external data sources, we have used our data model named "STriDE" (Semantic Trajectories in Dynamic Environments) to identify semantic points (POIs), semantic lines, and semantic regions (RoIs) in the trajectory data. Using this model, a system is designed to generate semantic trajectories in a dynamic environment for visualizing site-zone density to avoid congestion, proximity analysis to prevent workers' collisions, identifying an unauthorized access to hazardous areas, and monitoring movements of workers and machinery for reducing the transportation accidents.

The paper is organized as follows: in Section 2, the literature on semantic trajectories with existing semantic enrichment approaches is discussed. In Section 3, a system is presented to use semantic trajectories for an effective 
construction site monitoring and increasing the possibilities to carry safe construction operations by analyzing worker location data. Section 4 discusses the experimental analysis and the system benefits with some limitations, and Section 5 presents the conclusion with some future works.

\section{Background}

Existing literature reveals that the major reasons for fatalities on construction sites are linked to unsafe movements of the construction resources [10-12]. The technological advancements in mobile computing coupled with location-based services provide tremendous opportunities to monitor movements of construction resources to ensure safe construction operations [15-17]. The data acquired from a typical location acquisition device consists of a stream of spatio-temporal points [18]. This raw stream is cleaned by applying noise removing filters and transformed into finite meaningful episodes known as trajectories $[13,18]$. While the information from raw trajectories is useful to understand movement dynamics of objects in motion, it does not provide the contextual semantic information which is required to understand the meanings behind each trajectory episodes [14]. Existing studies $[7,19]$ have considered using location acquisition technologies along with contextual data repositories to construct spatio-temporal trajectories for tracking construction resources. However, to completely understand meanings behind user movements, spatio-temporal trajectories need enrichment with semantic data sources including application domain knowledge (building information) and geographic databases (such as OpenStreetMap (OSM) building file and Google Maps).

In the existing literature, there exist three major areas [13, 14] on semantic trajectories: trajectory construction, trajectory segmentation, and trajectory annotation [20] using semantic data sources. However, the focus of this research will be on the latter two areas. In general, there are two modes to construct a trajectory $[13,14]$ : (a) online mode, where trajectories are constructed in real time, and (b) offline mode, where all trajectories' construction processes are done in an offline mode. Although the literature on an online construction of trajectories is limited, there are many offline trajectory construction methods present in the literature [13]. In these methods, location data is collected in advance. Once location data is collected, it will undergo various processing stages such as data cleaning, map matching, and compression [13]. However, these methods are not suitable for real-life applications, where movements of objects are continuously updating. To address the requirement of an online trajectory construction for reallife applications, a real-time solution known as SeTraStream [21] is present having an ability to process raw trajectories data within a controlled time window and generate trajectories with start and end time in an online mode. Once trajectories are created, a process of segmentation is applied for dividing these trajectories into a set of episodes based on predefined criteria. The very first data model proposed by the authors [18], in which segmentation process is used to divide a trajectory into a set of moves and stops. A stop is defined as a place where a moving object has spent some specific time. However, other than a time threshold, segmentation can also be achieved by other attributes such as velocity, acceleration, direction, density, and geographic artifacts [22]. Similarly, there exists an extended segmentation framework to segment trajectories based on the movement states [23, 24]. However, their framework depends on the mapping of each movement state with relevant spatio-temporal criteria based on the expert knowledge, and manual user input. Moreover, Sankararaman et al. [25] presented an approach to distinguish between similar and dissimilar portions in trajectories. After then, trajectories are divided into segments to extract contiguous portions of trajectories which are shared by many of the other trajectories. Furthermore, segmentation can be done based on representativeness [26]. Such techniques perform global voting algorithms based on the local density and extract most representative sub-trajectories.

Once the segmentation of trajectories is completed, annotation techniques are applied to transform GPS trajectories into semantic trajectories [14]. The annotation process involves enrichment of trajectory episodes with the meaningful information such as the mapping of the POIs that can be in the form of points, in the form of lines, or the geographical regions [27, 28]. There are many annotation approaches present in the existing literature as provided in Table 1. Wu et al. [29] have used historic social media data to map it with user trajectories to understand the purpose of the trip. Based on the location history, relevant words are extracted from the Twitter data according to the mobility records, and user interests are retrieved for visiting a specific location at a specific time. In addition, user activities have also been used to annotate raw trajectories [30]. However, to cover a larger pool of the POIs, there is a need for integration with more datasets to enable tracking in large cities for the extraction of user activities [30].

Nogueira et al. have developed a framework to annotate trajectories based on episodes [31]. It basically defines the environment where the user trajectory has been taken placed based on the Linked Open Data (LOD) cloud and an OSM data. The ability of this framework of describing the spatial context of GPS trajectories can be used as a building block of future expert systems for trajectory exploration. Moreover, trajectories of slow- and fast-moving objects are also annotated at different levels of data abstraction using a multi-layer framework [27]. The locations where objects move provide information about their interests. At the same time, such behavioral analysis gives information about the popularity of visited places. A similar research effort is presented by 
Table 1 Comparison of existing semantic annotations of trajectories

\begin{tabular}{|c|c|c|c|c|c|c|c|c|}
\hline \multirow[t]{2}{*}{ Use case } & \multirow[t]{2}{*}{ Environment } & \multirow[t]{2}{*}{ Findings } & \multirow{2}{*}{$\begin{array}{l}\text { Key technologies/ } \\
\text { methods used }\end{array}$} & \multirow[t]{2}{*}{ Type of data } & \multicolumn{4}{|c|}{ Annotation types comparison } \\
\hline & & & & & $\begin{array}{l}\text { Social } \\
\text { media }\end{array}$ & Point & Region & Line \\
\hline $\begin{array}{l}\text { Semantic annotation of } \\
\text { mobility data using } \\
\text { social media [29] }\end{array}$ & Outdoor & $\begin{array}{l}\text { Extracted purposes and interests } \\
\text { of a user from his location } \\
\text { history. }\end{array}$ & $\begin{array}{l}\text { Kernel density } \\
\text { estimation model }\end{array}$ & $\begin{array}{l}\text { Geo-tagged } \\
\text { tweets }\end{array}$ & $\mathrm{Y}$ & $\mathrm{Y}$ & $\mathrm{N}$ & $\mathrm{N}$ \\
\hline $\begin{array}{l}\text { Inferring human activities } \\
\text { from GPS tracks [30] }\end{array}$ & Outdoor & $\begin{array}{l}\text { Automatically annotated } \\
\text { trajectories based on user } \\
\text { activities. }\end{array}$ & Gravity law & $\begin{array}{l}\text { GPS trajectories } \\
\text { of a car }\end{array}$ & $\mathrm{N}$ & $\mathrm{Y}$ & $\mathrm{N}$ & $\mathrm{N}$ \\
\hline $\begin{array}{l}\text { Annotating semantic } \\
\text { trajectories based on } \\
\text { episodes [31] }\end{array}$ & Outdoor & $\begin{array}{l}\text { Environments are identified } \\
\text { where trajectories took place. }\end{array}$ & $\begin{array}{r}\text { Linked Open Data } \\
\text { cloud and OSM }\end{array}$ & $\begin{array}{l}\text { GPS trajectories } \\
\text { of a jogger }\end{array}$ & $\mathrm{N}$ & $\mathrm{N}$ & $\mathrm{Y}$ & $\mathrm{N}$ \\
\hline $\begin{array}{l}\text { Semantic annotation of } \\
\text { heterogeneous } \\
\text { trajectories [27] }\end{array}$ & Outdoor & $\begin{array}{l}\text { Annotated trajectories for any } \\
\text { kind of moving objects. }\end{array}$ & Java 6 (PostgreSQL) & $\begin{array}{l}\text { GPS records of } \\
\text { taxis and } \\
\text { private cars }\end{array}$ & $\mathrm{N}$ & $\mathrm{Y}$ & $\mathrm{Y}$ & $\mathrm{Y}$ \\
\hline $\begin{array}{l}\text { Automated semantic } \\
\text { trajectory annotation } \\
\text { with indoor POI visits } \\
\text { [32] }\end{array}$ & Outdoor & $\begin{array}{l}\text { Combined multiple trajectory } \\
\text { pre-processing techniques to } \\
\text { extract POIs. }\end{array}$ & - & $\begin{array}{l}\text { Trajectories } \\
\text { formation } \\
\text { using uploaded } \\
\text { pictures }\end{array}$ & $\mathrm{N}$ & $\mathrm{Y}$ & $\mathrm{N}$ & $\mathrm{N}$ \\
\hline $\begin{array}{l}\text { Automated semantic } \\
\text { annotations based on } \\
\text { existing knowledge } \\
\text { bases [33] }\end{array}$ & Outdoor & $\begin{array}{l}\text { Abstraction in a multilevel } \\
\text { hierarchy of progressively } \\
\text { detailed movement segments. }\end{array}$ & $\begin{array}{l}\text { geoSPARQL and } \\
\text { PostGIS }\end{array}$ & $\begin{array}{l}\text { User's trail from } \\
\text { Flickr and } \\
\text { tweets }\end{array}$ & $\mathrm{Y}$ & $\mathrm{Y}$ & $\mathrm{Y}$ & $\mathrm{Y}$ \\
\hline $\begin{array}{l}\text { Dynamic semantic } \\
\text { annotation of } \\
\text { trajectories [34] }\end{array}$ & Outdoor & $\begin{array}{l}\text { Annotation using contextual } \\
\text { social media data. }\end{array}$ & $\begin{array}{l}\text { Kernel density } \\
\text { estimation model }\end{array}$ & $\begin{array}{l}\text { Geo-tagged } \\
\text { tweets }\end{array}$ & $\mathrm{Y}$ & $\mathrm{N}$ & $\mathrm{N}$ & $\mathrm{N}$ \\
\hline $\begin{array}{l}\text { Mining semantic } \\
\text { trajectory mobility } \\
\text { patterns [35] }\end{array}$ & Outdoor & $\begin{array}{l}\text { Characterization of the semantic } \\
\text { mobility of vehicles is } \\
\text { achieved by tagging visit } \\
\text { purpose to each trajectory. }\end{array}$ & $\begin{array}{l}\text { Google Maps and } \\
\text { prefix span } \\
\text { algorithm }\end{array}$ & $\begin{array}{l}\text { Private vehicle } \\
\text { trajectories }\end{array}$ & $\mathrm{N}$ & $\mathrm{N}$ & $\mathrm{Y}$ & $\mathrm{N}$ \\
\hline $\begin{array}{l}\text { Finding semantic level } \\
\text { trajectory behaviors } \\
\text { through semantic } \\
\text { trajectory clustering } \\
{[36]}\end{array}$ & Outdoor & $\begin{array}{l}\text { Extracted common semantic } \\
\text { trajectories using an extended } \\
\text { OPTICS algorithm. }\end{array}$ & $\begin{array}{l}\text { Density-based } \\
\text { clustering } \\
\text { algorithm }\end{array}$ & $\begin{array}{l}\text { Geo-tagged } \\
\text { photos from } \\
\text { Flickr }\end{array}$ & Y & $\mathrm{N}$ & $\mathrm{Y}$ & $\mathrm{N}$ \\
\hline
\end{tabular}

Graaff et al. [32], where an algorithm is proposed for combining existing multiple trajectory pre-processing methods to identify the visited POIs for detecting different indoor activities in an urban setting. Furthermore, the ontological modeling approach has also been used to abstract trajectory data in a multilevel hierarchy using LOD collections and social media data [33]. This integration can enable to query trajectories for mobility analysis based on the domain and application-related knowledge. In addition to automatic semantic annotations, there also exist dynamic and clustering-based semantic annotation methods based on the contextual and geo-localization data in the literature [34-36]. Such methods calculate the local density of words and map words to each trajectory record, hence providing visualizations for trajectories exploration.

After performing an extensive study on semantic trajectories, some research gaps have been realized in the existing literature before constructing a semantic trajectory system for safety management of workers on construction sites. These gaps encompass (1) existing semantic trajectory enrichment models are designed specifically for outdoor environments for tagging the relevant semantic information with the moving persons or vehicles' trajectories. The extraction of the insights related to the behaviors of moving objects within the building settings is not yet adequately explored. (2) To the best of our knowledge, the data models present in the literature for performing semantic enrichments hold static information regarding the environment in which the objects are in motion. However, for modeling reallife trajectories and extracting real-time insights about the moving object behaviors, an updated contextual information related to the building environment is necessary by the trajectory data model to generate semantically enriched trajectories. (3) The focus of the baseline models presented in Table 1 has been kept limited only to construct semantic trajectories. The feasibility of visualizing the semantic trajectories by integrating the trajectory data models' output with the existing open-sourced smart city solutions, for example, building information modeling (BIM) for different industrial application scenarios is missing in the literature. 


\section{Semantic enrichment for worker trajectory (SEWoT) system}

To fill the above-mentioned research gaps, a semantic trajectory processing, a visualization system named "SEWoT," is developed to monitor worker interactions remotely and to identify their abnormal movement behaviors. For constructing the "SEWoT" system, a scenario-based methodology [37, 38] is adopted by taking two roles into account which are as follows: building supervisor and H\&S manager. A scenario defines our system as a sequence of events which includes the following: (1) collecting location data from handheld devices of workers on a construction site, (2) constructing trajectories after performing pre-processing techniques, and (3) enriching them with the relevant semantic information extracted from an OSM data file, user-defined taxonomies, and contextual building information for building supervisors and H\&S managers to improve worker safety by visualizing the semantic trajectories as shown in Fig. 2.

Based on the developed scenario, a prototype system is designed for studying the user movements inside the building for an experimental analysis. However, the functionality of the prototype system will remain the same if deployed on a construction site. For capturing the location data of the building users, Bluetooth beacons are placed in different building locations. The collected location coordinates are pre-processed after removing the outliers and stored in a document database (MongoDB). Stay points of a user are extracted by inputting time and distance threshold values to identify the building locations where the user is spending significant time. These thresholds for calculating stay points are completely adjustable according to the application requirements. The extracted stay locations are critical for the analysis as their extended or short stay behavior as compared to the required will indicate the occurrence of undesired events in the building. Later, these stay points are tagged with their corresponding spatial information using our STriDE model that has a capability of tracking the dynamic environment for enriching the trajectories with relevant semantic information. After mapping the stay locations of a trajectory to semantic locations of a building, the moving segments within a trajectory is divided into the alternate walk and run segments to monitor the traveling speed of users. In the end, to visualize an entire trajectory having stopped and move segments, and with identified stay locations, a trajectory is mapped with a spatial region where it has occurred. In our case, the trajectory was captured from an indoor environment and tagged as a "work-zone." However, construction sites typically divided into many areas

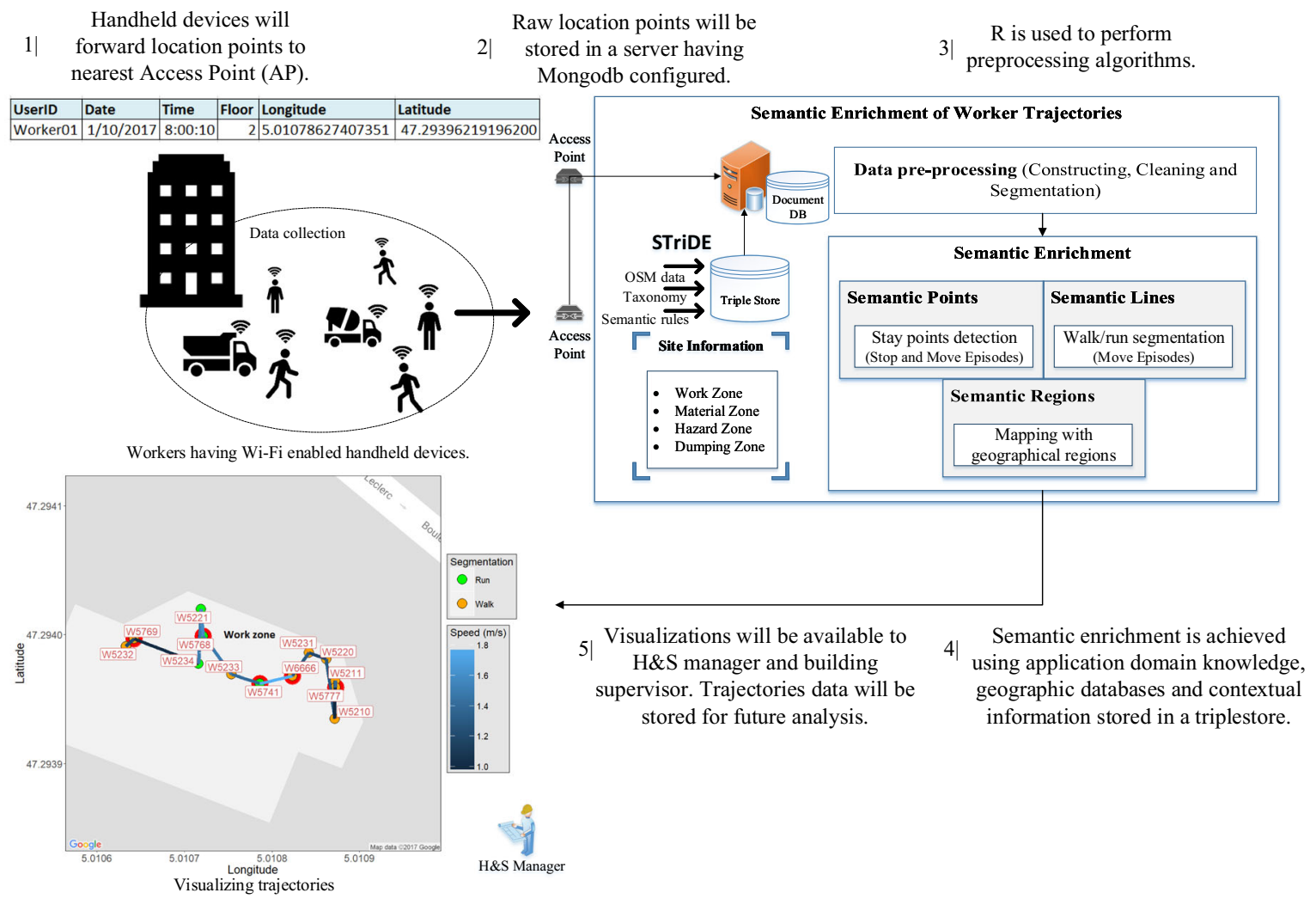

Fig. 2 Scenario for SEWoT system for worker movement analysis 
such as hazard-zone, material-zone, and dumping-zone by the building supervisors for site management [20]. In the end, benefits of semantic trajectories generated by our developed prototype system are discussed. The processes of our prototype system which are mentioned above are described in detail below.

\subsection{Trajectory data collection}

To collect trajectory data of users, 200 Bluetooth beacons [39] are placed in different locations in the building. Each beacon is detectable by the Wi-Fi-enabled handheld device within the radius of $4 \mathrm{~m}$. To acquire location coordinates, a mobile application using the Android platform is developed for detecting beacons and performing triangulation technique to get a unique pair of building location coordinates having longitude, latitude, and the floor number values. The process of tagging is achieved by utilizing the stored spatial information residing in a database as a deployment map of the beacons. Using this method, 13,223 location points are recorded across different locations with a sampling interval of $5 \mathrm{~s}$. However, the mobile application has the capability to set different sampling interval ranges from 0.5 to $5 \mathrm{~s}$. An application programming interface is used to capture the location data through wireless access points, aggregating and then storing it in a document-oriented database such as MongoDB. A data link is configured between a database system and $\mathrm{R}$ studio to pre-process the captured location data. There exist many noise filters, such as mean and median filters to improve the data quality. However, a median filter is used on the acquired data (as shown in Fig. 3) because it depicts robustness property in filtering and recommended for data with high sampling rate whereas, mean filter is not recommended because it is highly sensitive to outliers [13].

\subsection{Stay points detection for identifying POls}

After removing the outliers in a trajectory data, stay points of a user are calculated to enrich a user trajectory with semantic points in the form of stop and move segments. Stay points carry more important semantic information than moving points as these are the location points where a user has spent a significant time within a specified distance. By manually setting the distance threshold value $\left(D_{\text {thresh }}\right)$ to $5 \mathrm{~m}$ and time threshold $\left(T_{\text {thresh }}\right)$ value to $20 \mathrm{~min}$, stay points in a trajectory are identified (as shown in Fig. 4) using Zheng et al. [13] approach. The existing literature $[40,41]$ specifies a $T_{\text {thresh }}=$ $10 \mathrm{~min}$ and $D_{\text {thresh }}=50$ to $250 \mathrm{~m}$ for identifying user stay locations in an outdoor environment. However, inputting a $T_{\text {thresh }}=10 \mathrm{~min}$ in our system resulted in many redundant stay locations. Therefore, we doubled the value of a $T_{\text {thresh }}$ and set $T_{\text {thresh }} \geq 20 \mathrm{~min}$ for extracting the locations where the user has spent a significant time. In addition, existing works [40, 41] only contain the case studies for extracting the stay locations in outdoor environments where the locations are on the greater distances from one another. For an indoor environment and taking the dimensions of the building rooms into consideration, we set the value of $D_{\text {thresh }}$ to $5 \mathrm{~m}$. The justification of using these thresholds is presented in the Section 5. Here, an extracted single stay point"s" [13] can be treated as a virtual location point characterized by a set of successive GPS points $Z=\left\{z_{m}, z_{m+1}, z_{m+2}, \cdots, z_{n}\right\}, \forall m<i \leq n$, Distance $\left(z_{m}, z_{i}\right) \leq$ $D_{\text {thresh }}$, and $\left|z_{n} . T-z_{m} . T\right| \geq T_{\text {thresh. }}$. A stay point can be described as $s=$ (Latitude, Longitude, arrivaltime, leavingtime).Where,

$$
\begin{aligned}
s . \text { latitude } & =\sum_{i=m}^{n} z_{i} \text {. Latitude } /|Z| \\
s . \text { lontitude } & =\sum_{i=m}^{n} z_{i} \text {. Longitude } /|Z|
\end{aligned}
$$

The purpose of calculating stay points in a trajectory data is to find locations in a building where users are spending more time than required. This information will help to track the occurrence of an unexpected situation on a site if stay duration is greater or less than the required. The stay duration can be adjusted as per the application requirement as having smaller threshold values for distance and time will lead to several stay points whereas, larger threshold values will result in fewer points. An assumption that is considered for verifying the stay points is visualizing the spatial density of location points in the trajectory data [42]. According to Palma et al. [42], the moving object speed decreases considerably when a place is visited. However, the data acquisition system will keep collecting location points according to its configuration while an object
Fig. 3 Filtered raw trajectory data of a user

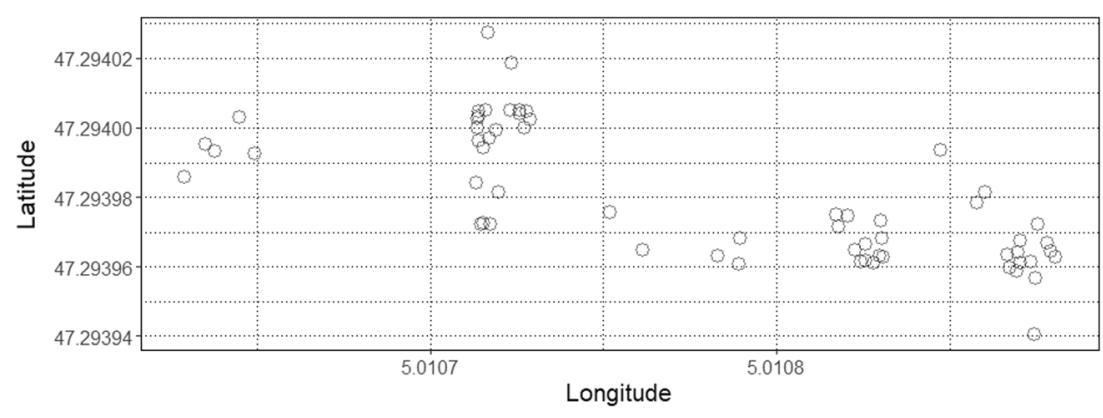


Fig. 4 Stay point extraction with time spent

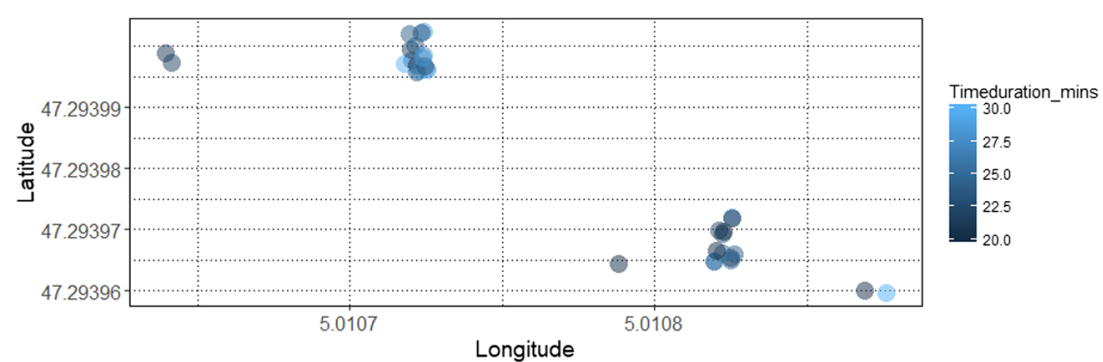

is stopped that will result in the greater spatial density of location points that is visible in Fig. 4.

\subsection{SEWoT system for visualizing semantic points, semantic lines, and semantic regions}

Once stay points (POIs) are identified, these points are annotated with the corresponding semantic information to achieve semantic points. For enriching user trajectories using semantic data sources, a data model named STriDE [43] is used. The STriDE model as shown in Fig. 5 is built to store semantic trajectories by addressing the needs of a dynamic environment where the building objects (user, trajectory, and location) can move or even change their geometry (shape and size) or attributes (alphanumeric semantic information) with time. For tagging the different building location with the processed trajectory data, the STriDE model uses the "concepts." As a worker location changes in a building, there will be a change in the type of "concept" labeled with its trajectory point. However, the labeling of the "concept" will not be done directly with the trajectory point but with its timeslice as shown in Fig. 5. A timeslice has four components: an identification, alphanumeric attributes, a time component indicating the validity of a timeslice, and a geographical component depicting the spatial representation of an entity [43]. With the help of timeslices, if there is any change detected in the geometry or semantic information related to the building entities (user, trajectory, and location), a new timeslice will be created with updated information and linking it with the last known timeslice. This mechanism will help in storing information about the evolution of the building structure during its lifecycle for generating trajectories with the most updated semantic information.

For constructing semantic trajectories, STriDE model is fed with an IFC (Industry Foundation Classes) or OSM (OpenStreetMap) file, annotation rules, and taxonomies. In the prototype system, the application of an OSM file is to manage a complete building structure using the geographic vector data in an Extensible Markup Language (XML) format in which rooms' boundaries are defined with their links to each other. As shown in Fig. 6, an OSM file contains nodes, ways, and relations. Entities in an OSM file can be labeled with single or multiple key-value pairs to add the semantic information. For instance, in Fig. 6, a "way" having an ID = 235 is specified as a corridor by adding a tag "highway = corridor" that relates to a collection of nodes' references such as 2755,2756 , and 2757 corresponding to different longitude and latitude values. These labeled key-value pairs are grouped and managed using taxonomies which are created by the domain experts. A taxonomy is a hierarchy of concepts written as RDF (Resource Description Framework) triples. For instance, in Fig. 7, a scheme of concepts named "ElementScheme" is created containing a top concept (root) named "Element." This element has a narrower concept named "Path," which itself has a narrower concept named "Corridor." Moreover, annotation rules are created using a JavaScript Object Notation (JSON) file structure for linking OSM objects with the taxonomy. For example, an annotation rule described in Fig. 8 shows that any OSM entity carrying a tag with a key "Highway" and having a value "Corridor"
Fig. 5 STriDE data model representing dynamic entities (a user, a trajectory, and a room) each with a timeslice (TS). Each TS contains the geometry, alphanumeric properties (semantics), and a time span for which it is valid. Whereas, the concepts are used for mapping the semantic locations for user profiling and access control

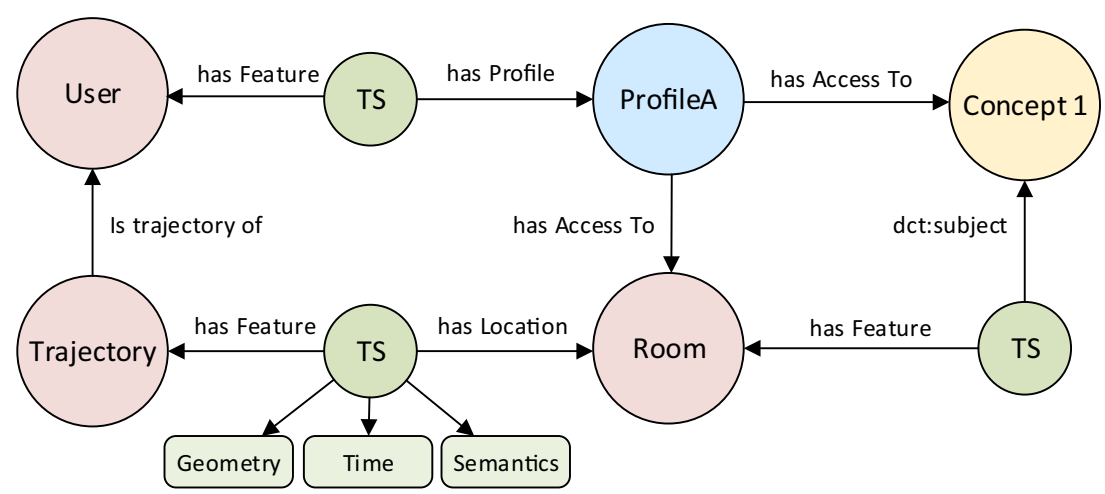




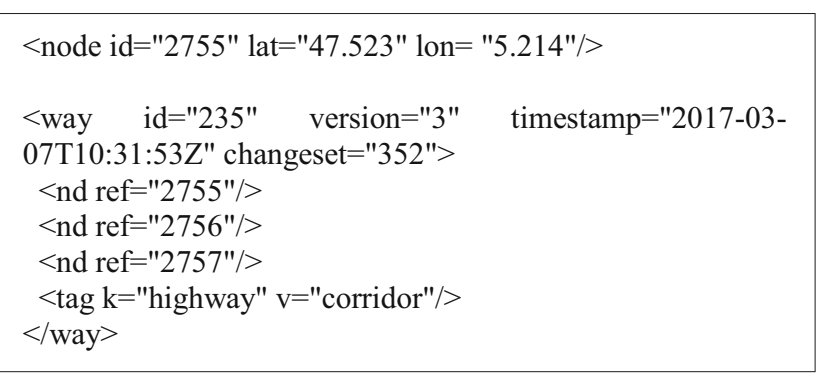

Fig. 6 An OSM file record in which a "way id" $=235$ is defined using a collection of nodes references that are 2755, 2756, and 2757 corresponds to unique pairs of longitude and latitude values

should be labeled with a concept "Corridor," i.e., https://www. u-bourgogne.fr/stride\#Corridor. Later, an OSM file with annotation rules is inputted to a 2-step Java parser. The parser will create mappings between OSM entities with Java objects and then constructed Java objects are transformed into new Java objects as per the semantic definition. Finally, the processed Java objects (see Fig. 9) are stored in a triplestore (Stardog) for attaining the complete representation of the building structure.

Using the contextual and geographical information (see Table 2) which is stored in the STriDE model (see Fig. 9), the mapping of trajectory episodes to the meaningful information such as the mapping of places of interest that can be in the form of POIs (e.g., W5768) and the ROIs (e.g., work-zone) is achieved. The mapping of the POIs and ROIs with the trajectory points is performed using a topological correlation, i.e., Trajectory_epidode $\bowtie_{\theta}$ Location. Here, the parameter $\theta$ is computed using the topological spatial relations such as distance and displacement [27, 28]. After computing the value of $\theta$,

\footnotetext{
@prefix skos: <http://www.w3.org/2004/02/skos/core\#> . (a) prefix stride: <https://www.u-bourgogne.fr/stride\#> . stride:ElementScheme a skos:ConceptScheme ;

skos:prefLabel "Thesaurus of the elements of a building"@en ; skos:hasTopConcept stride:Element .

stride:Element a skos:Concept ;

skos:prefLabel "Element"@en ;

skos:inScheme stride:ElementScheme .

stride:Path rdfs:type skos:Concept ;

skos:prefLabel "Path"@en ;

skos:broaderTransitive stride:Element ;

skos:inScheme stride:ElementScheme

stride:Corridor rdfs:type skos:Concept ;

skos:prefLabel "Corridor"@en ;

skos:broaderTransitive stride:Path ;

skos:inScheme stride:ElementScheme
}

Fig. 7 Taxonomy (the script is a RDF Turtle https://www.w3.org/TR/ turtle/ of the concept "Corridor" in the partially extracted STriDE schema. A scheme of concepts named "ElementScheme" is created having a top concept (root) named "Element." This element has a narrower concept called "path," which itself has a narrower concept called "corridor")

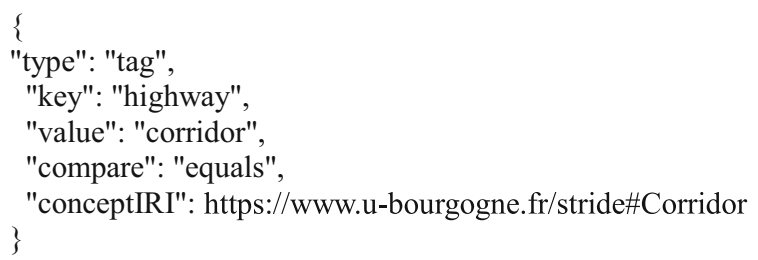

Fig. 8 Annotation rule showing any OSM entity having a tag whose key is "highway" and whose value is "corridor" is to be tagged with the concept "corridor," i.e., https://www.u-bourgogne.fr/stride\#Corridor

spatial joins are performed with the boundary of the trajectory. In this way, a location with its associated semantic metadata is annotated with the trajectory's episodes. This process is used for finding the ROIs in the trajectory data. In the same way, a list of POIs exists inside the building which are the "workspaces" (W5769, W5768, W6666, W5741, and W5777); in our case, they are also defined in the STriDE model. Using this stored semantic information, each stay location is tagged with the site identification (ID) that corresponds to different site regions (ROIs) such as work-zone, material-zone, hazard-zone, and dumping-zone. Construction sites typically have more work zones [7], but we have restricted site zones to four for this research. After tagging the semantic points, successive stay locations are grouped together, and aggregated stay duration in minutes is calculated to remove repetition and to reduce the size of a trajectory (see Figs. 10 and 11).

We can now enrich a trajectory with semantic lines in the form of movement types which are being carried by workers after mapping the spatio-temporal locations in a trajectory to corresponding site identifications. Based on our application requirement, we have used walk-based segmentation method of Zheng et al. that is based on semantics 5. Walk points and run points based on points' speed values are calculated, then a trajectory is divided into an alternate walk and run segments as

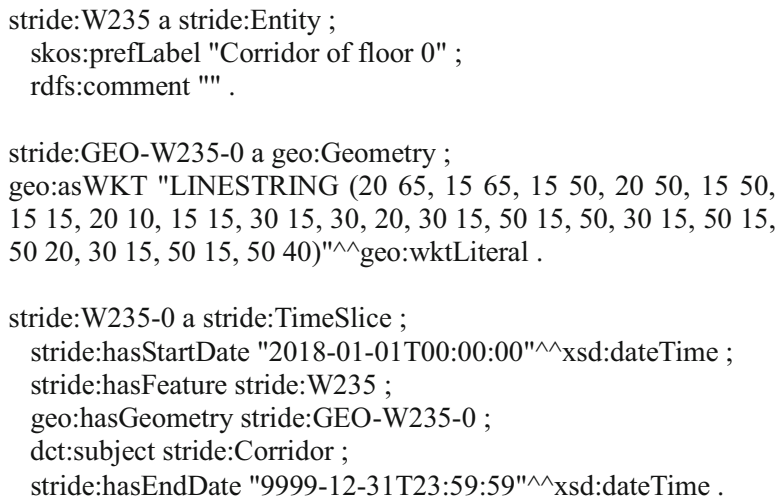

Fig. 9 Parsed OSM file using the annotation rules and the taxonomy (the script is the RDF Turtle definition of an object of the kind "Corridor" identified by the value stride:W235. The object holds its name, a geometry defined by a set of latitude and longitude pair values and a timeslice having start and end timestamps for its validity. These values are used to define semantic trajectories) 
Table 2 Mapping of IPS data with the construction site/building information

\begin{tabular}{lllll}
\hline GPS coordinates [Lon, Lat] & Geometry type & Semantic Region (ROI) & Semantic Point (POI) & Building name \\
\hline $\begin{array}{c}\{5.010756,47.293998],[5.010729,47.294025], \\
[5.010765,47.294017],[5.010756,47.293998]\}\end{array}$ & Polygon & Work-zone & W5768 & Venue213 \\
\hline
\end{tabular}

shown in Fig. 6a. Moreover, speed is also calculated between two segments in meters per second. Lastly, to visualize the entire trajectory having stop and move segments with a semantic region, $\mathrm{R}$ studio is used with Google Maps as shown in Fig. 6b. After mapping the spatio-temporal locations in a trajectory to the site/building identifications, we can now enrich a trajectory with semantic lines in the form of movement types which are being carried by a user. We have used walk-based segmentation method of Zheng et al. [13] which is based on semantics. Walk points and run points based on points' speed values are calculated, and a trajectory is divided into an alternate walk and run segments as shown in Fig. 12. Moreover, speed is also calculated between each successive segments of a trajectory in meter per second. Lastly, to visualize the entire trajectory having stop and move segments with a semantic region (i.e., Venue213), R studio is used to establish the topological correlations using spatial joins between a processed trajectory and a spatial region information extracted from the STriDE model to identify the actual building as shown in Fig. 13.

\subsection{Comparative analysis}

Comparing data modeling approaches is a subjective task [31]. In this section, we have compared our developed system with the existing semantic enrichment systems as shown in Table 3. Identification of the locations, clustering of trajectories based on the similar behaviors, reducing trajectories for visualizations, trajectory segmentation for dividing trajectories into stop and move episodes, transportation mode detection, predicting the next locations of the users, recommendation based on the previous trajectory data logs, activity recognition based on the visited locations, mapping with the environmental information, and behavior categorization have been the major characteristics of the existing semantic enrichment systems. After this review, an idea of the basic functionalities of a typical semantic enrichment system is conceived from these state-of-the-art applications for the development of our proposed system. It is observed that the potential of semantic trajectories for improving the safety of construction resources in dynamic environments is not well explored in the studied literature, and very little research has been found regarding this area. The present trajectory data models are designed specifically for outdoor environments and the extraction of the behaviors of moving objects within the building using semantic trajectories is not yet adequately explored. In addition, the existing trajectory models do not cater the data modeling requirements for dynamic environments where the building objects are moving or changing with time. Moreover, the baseline models as mentioned in Table 3 do not show the possibility of integrating the trajectory data models' output with the existing open-sourced deployed smart city solutions (for example, BIM) for the industrial users. Our developed trajectory processing and visualization platform offers a new application of semantic trajectories for construction safety management which holds a potential to help H\&S managers in their dayto-day building operations using the industry's open standard software (i.e., BIM) in monitoring user-buildings interactions remotely in outdoor as well as in indoor dynamic environments and to take timely actions in real time in case of abnormal behaviors found in worker trajectories.

\section{Experimental analysis}

The developed "SEWoT" system for worker movement analysis is validated using a real-life IPS feed of the building users. Table 4 shows a summary of the data used in our experimental analysis. 13,223 IPS records of 13 building users are collected

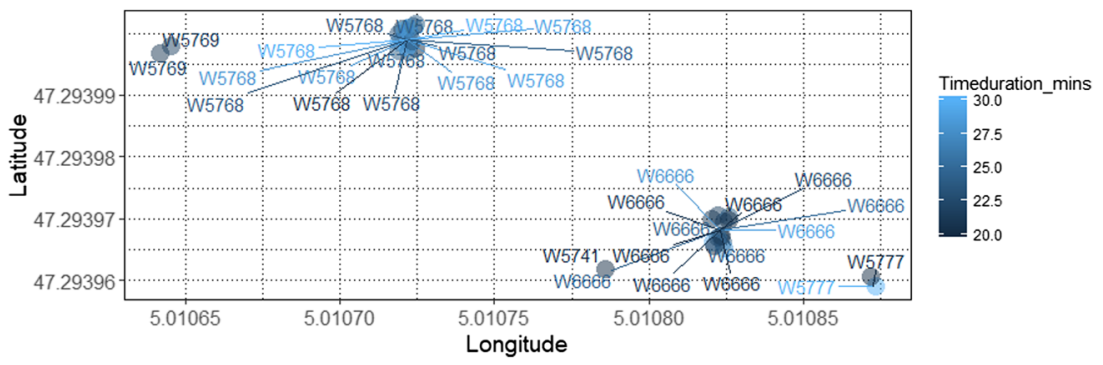

Fig. 10 Semantic enrichment of stay points with site location IDs (stay locations which correspond to the semantic locations of a building named as W5769, W5768, W6666, W5741, and W5777 respectively. These locations are detected by setting the time threshold $\left(T_{\text {thresh }}\right) \geq 20 \mathrm{~min}$ and the distance threshold $\left(D_{\text {thresh }}\right)=5 \mathrm{~m}$. The lighter the color of a stay point depicts the shorter stay duration of the user 
Fig. 11 Visualization of semantic stay points with stay durations calculated in minutes. (Successive stay points of the user are grouped for calculating the total stay duration at a building location. The stay duration ranges from 25 to $125 \mathrm{~min}$ at the locations named as W5769, W5768, W6666, W5741, and W5777)

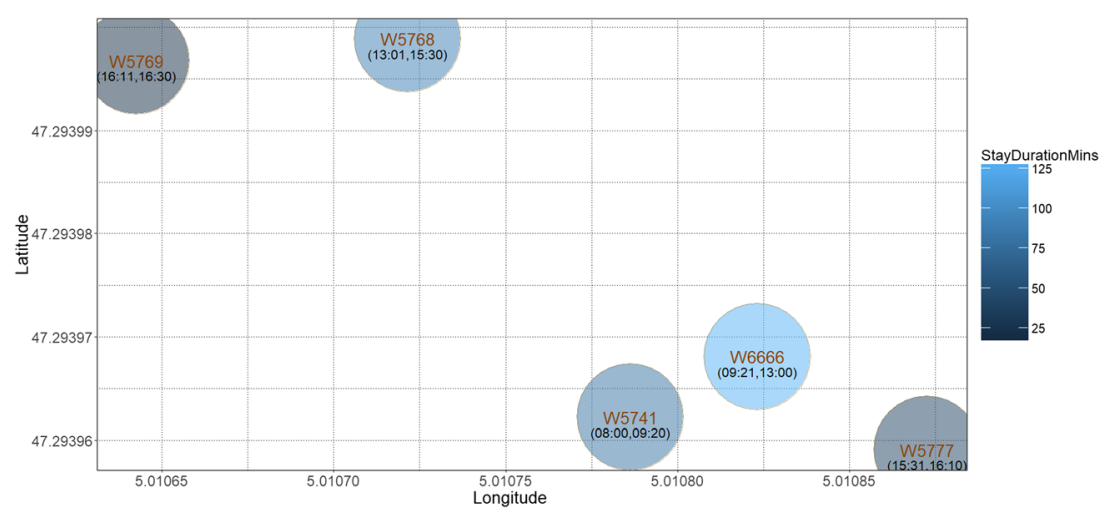

during different intervals in a 2 -week period with a sampling frequency of $5 \mathrm{~s}$.

For developing our prototype system, we have used the R platform for data processing and a Stardog for storing semantic information. Using the STriDE model, which holds the information of 328 semantic locations of a building extracted using an OSM file, is utilized for the semantic enrichment process. Figures 3, 4, 5, 6, 7, 8, 9, 10, 11, 12, and 13 show a process of transforming raw IPS feed to semantically enriched trajectories. Based on the existing literature $[40,47]$ which covers the matrices to evaluate the classification systems, we have assessed the efficiency of our developed semantic enrichment system against different values of distance and time thresholds for extracting the stay locations (POIs) to map them with their corresponding semantic information (Table 5).
An interesting aspect to observe here is that as we increase the values of time and distance thresholds, our model mapping precision of tagging semantic locations increases. However, the higher values of inputting time and distance thresholds will result in the identification of fewer stay locations in the trajectory data of a user because some of the stays join together. Whereas, if the smaller values of time and distance threshold are used, it resulted in overlapping in the tagging of the semantic locations and the exact locations are not tagged effectively. The stay point detection thresholds cannot be adjusted dynamically but should be set as per the application requirements. Ultimately, we have used $T_{\text {thresh }}=20 \mathrm{~min}$ and $D_{\text {thresh }}=5 \mathrm{~m}$ for the prototype development to cover most of the stay locations of the user.

After mapping the semantic information related to the building environment with the detected stay locations, walk
Fig. 12 Segmenting a worker trajectory in the run and walk segments to achieve semantic lines (the speed of a user is calculated in meter per second $(\mathrm{m} / \mathrm{s}))$. Value of speed is calculated between succeeding semantic locations. Based on the calculated speed values, the segments having the walk speed $\leq 1.4 \mathrm{~m} / \mathrm{s}$ are shown as "walk segments" in "orange" color. Whereas, the segments having the walk speed $>1.4 \mathrm{~m} / \mathrm{s}$ are shown as "run segments" in "green" color

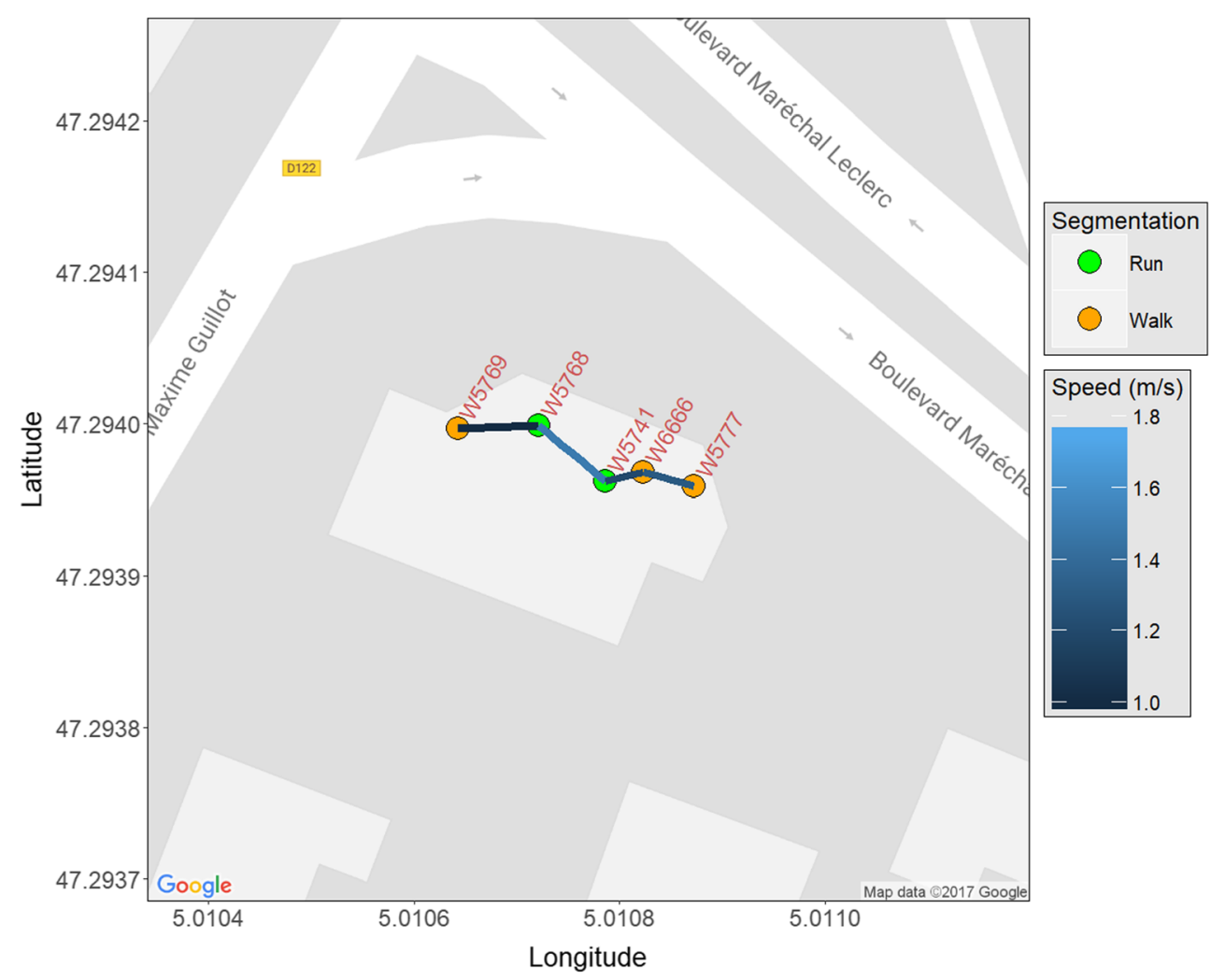


Fig. 13 Visualizing a complete user trajectory on a building model with semantic points in red colored circles (site

identifications, e.g., W5768 for a work-zone), semantic lines (segmentation into run and walk points) and a semantic region (work-zone)

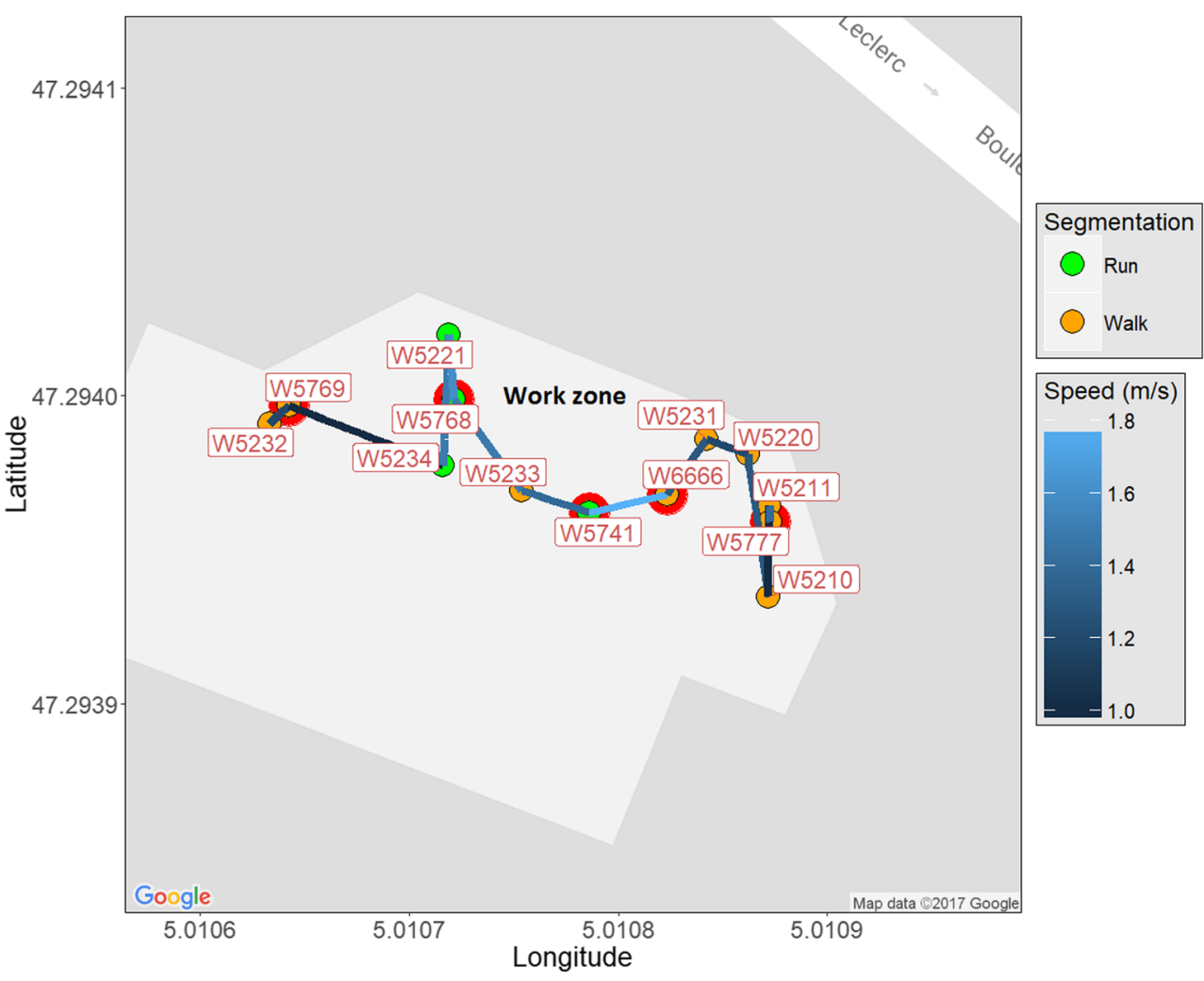

and run segments (semantic lines) are constructed using the spatio-temporal attribute (speed value) and finally enrichment with the semantic region for mapping a complete trajectory on an actual building location is done for understanding the user movements. The developed system will serve not only as a semantic trajectory visualization platform for safety managers but also acts as a data repository of all types of movements occurring on sites for future hazard analysis. The analysis of the generated semantic trajectories will help to achieve belowmentioned benefits.

- Visualizing site-zones' density to avoid congestion: Workforce on a construction site is almost half of the project's cost, and it is important to improve logistics associated with their management on sites [48]. To ensure that workers are working in good conditions, space planning
Table 3 Comparison of existing trajectory models

\begin{tabular}{|c|c|c|c|c|c|c|c|c|c|c|c|c|}
\hline \multirow[t]{2}{*}{ Model } & \multicolumn{12}{|c|}{ Purpose* } \\
\hline & A & $\mathrm{B}$ & $\mathrm{C}$ & $\mathrm{D}$ & $\mathrm{E}$ & $\mathrm{F}$ & $\mathrm{G}$ & $\mathrm{H}$ & $\mathrm{I}$ & $\mathrm{J}$ & $\mathrm{K}$ & $\mathrm{L}$ \\
\hline MADS [18] & & & & $\mathrm{Y}$ & & & & & & & & \\
\hline SeMiTri [21] & $\mathrm{Y}$ & $\mathrm{Y}$ & & $\mathrm{Y}$ & $\mathrm{Y}$ & & & $\mathrm{Y}$ & & $\mathrm{Y}$ & & \\
\hline The Baquara [44] & $\mathrm{Y}$ & & & $\mathrm{Y}$ & $\mathrm{Y}$ & & & $\mathrm{Y}$ & $\mathrm{Y}$ & $\mathrm{Y}$ & & \\
\hline CONSTAnT [45] & $\mathrm{Y}$ & & & $\mathrm{Y}$ & $\mathrm{Y}$ & & & $\mathrm{Y}$ & $\mathrm{Y}$ & $\mathrm{Y}$ & & \\
\hline The Baquara $^{2}$ [33] & $\mathrm{Y}$ & $\mathrm{Y}$ & $\mathrm{Y}$ & $\mathrm{Y}$ & $\mathrm{Y}$ & & & $\mathrm{Y}$ & $\mathrm{Y}$ & $\mathrm{Y}$ & & \\
\hline SemMobi [34] & $\mathrm{Y}$ & $\mathrm{Y}$ & & $\mathrm{Y}$ & & $\mathrm{Y}$ & $\mathrm{Y}$ & & & & & \\
\hline Unusual behaviors detection model [46] & $\mathrm{Y}$ & & & $\mathrm{Y}$ & & & & & $\mathrm{Y}$ & $\mathrm{Y}$ & & \\
\hline FrameSTEP [31] & $\mathrm{Y}$ & $\mathrm{Y}$ & & $\mathrm{Y}$ & $\mathrm{Y}$ & & & & $\mathrm{Y}$ & $\mathrm{Y}$ & & \\
\hline SMOPAT (Semantic MObility PATterns) [35] & $\mathrm{Y}$ & $\mathrm{Y}$ & & $\mathrm{Y}$ & & & & & & & & \\
\hline Proposed SEWoT system (this work) & $\mathrm{Y}$ & & $\mathrm{Y}$ & $\mathrm{Y}$ & & & & & $\mathrm{Y}$ & & $\mathrm{Y}$ & $\mathrm{Y}$ \\
\hline
\end{tabular}

$* A$, identification of locations; $B$, clustering of trajectories; $C$, trajectory reduction; $D$, trajectory segmentation; $E$, transportation mode detection; $F$, prediction; $G$, recommendation; $H$, activity recognition; $I$, mapping with the environmental information; $J$, behavior categorization; $K$, addressing dynamic environments; $L$, integration with the external systems for an industrial use 


\begin{tabular}{lllll}
\hline Dataset & No. of users & No. of IPS records & Tracking time & Sampling frequency \\
\hline Building users & 13 & 13,223 & 2 weeks & $5 \mathrm{~s}$ \\
\hline
\end{tabular}

on sites gets mandatory but often overlooked which leads to site congestion that can obstruct worker safety monitoring processes, and their productivity [48, 49]. Our system using IPS technology has made it possible to calculate the time spent by workers in each site area (see Fig. 11). For identifying critical site areas, worker stay locations are considered for the analysis as these locations are more crucial to monitor than the locations where workers are just passing through. With the visualization provided by our system, it establishes an understanding of how many workers are present in different areas of a site at a particular time and where they are spending the majority of their time. This information will help the building supervisors in determining most frequently visited stay locations, determining the appropriate number of workers to be in any specific site area by preventing site area congestion for safety management, and will also help them to monitor critical site areas' utilization throughout a day.

- Proximity analysis to prevent workers' collisions: According to the OSHA statistics [3], most fatalities occurring on construction sites are very closely related to the unsafe proximity of workers with the operating machinery, and the unsafe locations from where there is a risk of falling. Visualizing multiple semantic trajectories simultaneously generated by our system (see Fig. 14) in real time can help H\&S managers to keep track of the proximity of workers and machinery in real time. Our system uses a Haversine distance formula [39, 48] between two semantic trajectories to calculate distance in meters as shown below;

$$
d=2 \sin ^{-1}\left(\sqrt{\sin ^{2} \frac{x_{t+1}-x_{t}}{2}+\cos x_{t} \cos x_{t+1} \sin ^{2} \frac{y_{t+1}-y_{t}}{2}}\right)
$$

where " $r$ " is the radius of the earth and geographical coordinates (longitude and latitude) are represented as $x$ and $y$. The reason for using the Haversine distance formula is because it is one of the preferred methods that calculate the geographic

Table 5 Impact of time and distance thresholds on semantic enrichment process

\begin{tabular}{llll}
\hline $\begin{array}{l}T_{\text {thresh }} \\
\text { (minutes) }\end{array}$ & $\begin{array}{l}D_{\text {thresh }} \\
\text { (meters) }\end{array}$ & $\begin{array}{l}\text { Detected } \\
\text { stay } \\
\text { locations }\end{array}$ & $\begin{array}{l}\text { Tagged } \\
\text { semantic } \\
\text { points }\end{array}$ \\
\hline 10 & 3 & 150 & 136 \\
20 & 5 & 110 & 107 \\
30 & 7 & 40 & 38 \\
\hline
\end{tabular}

distance between two points on a sphere [50]. In addition, semantic points that have been categorized as unsafe locations set by the H\&S manager can be used in the identification of the workers that are working too close or within the unsafe locations from where there are the risks of falling.

- Identifying unauthorized access to hazardous areas: Controlling workers access to hazardous zones of a site is important because having an unauthorized worker presence in the dangerous space, for example, a high-voltage room can result in fatality $[6,51]$. Typically, workers are briefed before starting the work as well as professionally trained to handle the complexities associated with working in hazardous areas [52]. However, it is not easy to monitor every worker in verifying whether he is authorized to work in certain dangerous spaces. Keeping a security check with the help of the hired guard at the entrance of every hazardous area or such areas which may contain expensive machinery is not possible and economical. Our system (see Fig. 5) offers the ability to perform user profiling and executing an access control system with the help of "concepts." These "concepts" correspond to different semantic locations defined in the STriDE model. Visualizing the "concepts" tagged to worker trajectory points in the form of semantic locations will allow H\&S managers to identify when a hazardous site location is detected within the trajectory of an unauthorized worker. This information will deliver pro-active safety information which will not only prevent unauthorized workers from staying in the hazardous areas but also eliminates the need for manual security procedures.

- Monitoring movements of workers and machinery to reduce transportation accidents: Another important information that safety managers like to monitor is the traveling speed of the workers and machinery on site. For example, the walking speed of a person is usually less than $1.4 \mathrm{~m} / \mathrm{s}$ [13], whereas a person moving with a speed greater than $1.4 \mathrm{~m} / \mathrm{s}$ will be annotated as a run segment. Workers or machinery moving at a higher than specified speed threshold inside a site zone would be considered unsafe. This information from our semantic trajectories (see Fig. 12) will work as a leading indicator of worker safety and can help in the identification of the workers and equipment moving at a high speed [53].

These were some possible benefits of our developed prototype system. However, further work is required to be done to fuse data from other emerging technologies such as BIM with 
Fig. 14 Visualizing two trajectories for observing their proximity within the site locations id: W5710

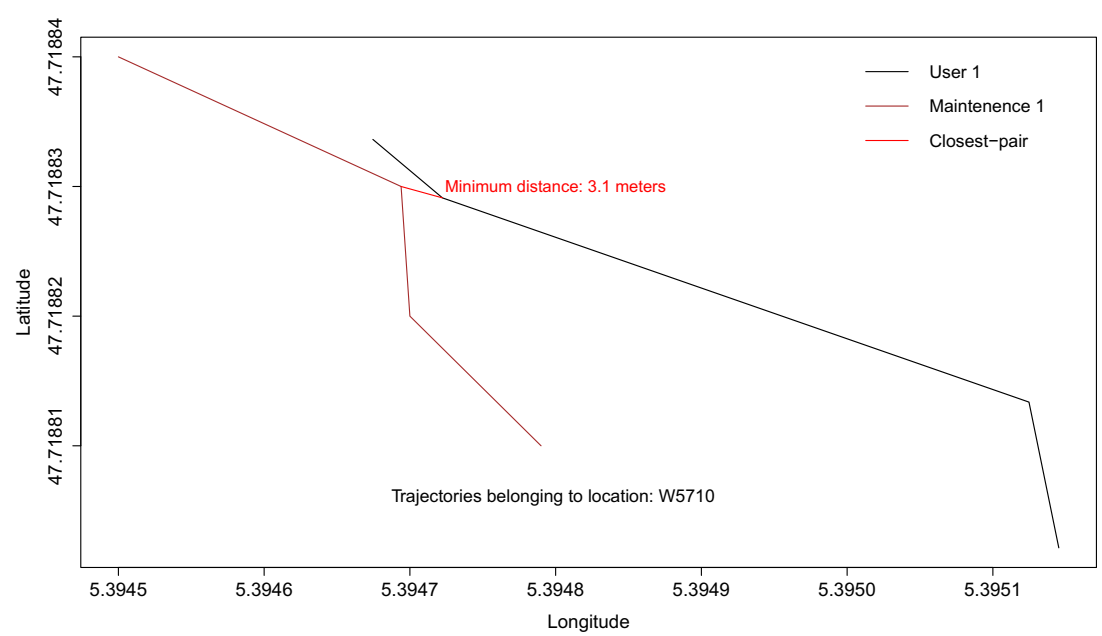

spatio-temporal trajectory data to visualize processed trajectories with the building infrastructure context. The BIM approach is proposed for generating visualizations after extracting insights from the semantic trajectories because literature identifies it as a "future IT solution" and preferred over traditional 3D CAD approaches as it is an efficient way of information management during the building lifecycle for safety analysis [54]. Above all, the BIM approach is becoming a construction industry standard in many countries [54]. An example of a BIM-based visualization for displaying semantic points and semantic lines (walk and run movements) is shown in Fig. 15.

In Fig. 15 above, an Autodesk Revit Architecture (a BIM software) is used. The BIM model of the building that is used for the study does not exist. A simple building structure without architectural esthetics is created in a Revit software utilizing the information from an OSM file of a building for demonstrating a proof-of-concept integration of systems. After constructing a BIM model of a building, all building spaces which are bounded by walls are tagged as "rooms" in the Revit software. Each tagged room contains the semantic information in the form of a set of parameters such as room number, room name, and physical area that can be used for viewing or editing that particular room. In our case, the parameter "room name" will act as a unique identification for each space representing a POI for visualizing the locations in the Revit model. The naming convention of Revit rooms is set according to the tagging of semantic points as described in our SEWoT system. Using a Dynamo (a Revit Plug-in) [55], semantic points and semantic lines are visualized in different colors on a BIM model. Figure 15 (left) is showing semantic trajectory information of a worker trajectory at time $t=1$. Whereas, Fig. 15 (right) is plotted for $t=2$. With the passage of time, the structure of a building has evolved because of the addition of new walls, while others are detached. These changes have resulted in different behavioral patterns (semantic points and semantic lines) of a worker as shown in Fig. 15. Investigating the reasons behind the change in the worker trajectory patterns requires previously stored semantic trajectory data along with the contextual building information (IFC or an OSM file). Our developed SEWoT system has the ability of keeping track the changes in the building environment using the STriDE model as discussed in Section 3.3. Resulted behavioral pattern analysis can be used for different construction and built environment applications such as construction resource monitoring for improved safety, managing
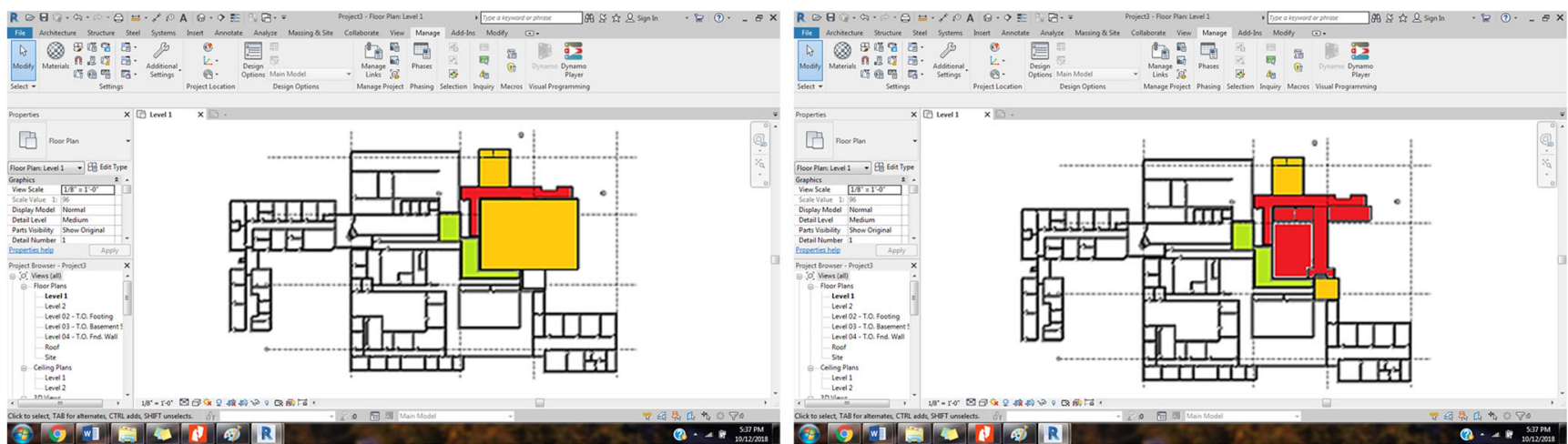

Fig. 15 BIM models constructed for showing semantic points of a worker in "orange," locations having "walk" trajectories in "green," and "run" trajectories in "red" colors 
building spaces based on their utilization, and implementing worker access control system. At present, the generated BIM visualization is static and does not have the capability for displaying updated trajectory insights in real-time. Constructing similar dynamic visualizations after addressing the needs of real-time trajectory data integration with the BIM software can be used by the H\&S managers in improving safety management intervention to prevent accidents by keeping track the locations where the workers are staying more than the required and identifying the locations where the workers are moving or operating the equipment at the high speed. In addition, further functionalities should be added in the system to incorporate pattern mining techniques to take complete advantage of fused information for deriving mobility behaviors of workers that will ultimately help safety managers for better decision making in safety processes.

Apart from the potential benefits and future directions to improve the system, there exist some limitations of the developed system. Bluetooth beacons are used to acquire the trajectory data. However, these beacons are not recommended for an indoor trajectory data acquisition system because of the less precision in the exactness in determining the building locations. As during the process of semantic enrichment, collected trajectory points did not totally join spatially with the information about the semantic points taken from an OSM file. Hence, the nearest possible semantic locations are tagged. To achieve high accuracy in trajectory data, an indoor geolocalization technology should be carefully chosen which should be robust to multipath fading and indoor noise. In addition, the experimentation is done using batch processing techniques [13] in which trajectories of building users in a day are first acquired and then pre-processing algorithms are implemented in an offline mode. However, for achieving the real-time insights of worker movements on construction sites, stream processing techniques [13] should be used to preprocess the worker trajectories in an online mode. So that trajectory multifaceted characteristics such as direction and speed can be computed as soon as location data is received for extracting trajectory insights.

\section{Conclusion}

Location is the crucial component of many processes and understanding the movement behaviors of users is getting more and more important. Monitoring the construction sites for movement behaviors of construction resources has always been a very challenging task because of the harsh environment and lack of technological infrastructure. Building supervisors and H\&S managers are in the need of real-time information about their resources such as workers and machinery to support their decision-making. This comprises accurate location data to understand worker occupancy in stay locations, types of their movements, and identification of unauthorized users on different site locations. Wireless local area networks (WLANs) are used extensively for estimating the location of users and providing a convenient as well as the cost-efficient method to track users in indoor as well as in outdoor settings. Moreover, smart handheld devices are becoming important in daily life, making it easier to be tracked by the deployed WLANs. Using this existing platform, having the WLANs and handheld devices as a sensor network, and by deploying Bluetooth beacons, a study is presented to capture location data of building users to study worker movement behaviors. Firstly, spatio-temporal trajectories are constructed using realtime location data of the building users. Secondly, locations of the users are extracted where the users have stayed for a longer time period. Thirdly, stop and move episodes of user trajectories are mapped with associated semantic information. It can be concluded from the visualizations generated of semantically enriched user trajectories that it provides a method to get information about (1) the occupancy of important site zones which are identified as stay locations of users, (2) proximity of workers and machinery to avoid collisions, (3) controlling unauthorized access of users to hazardous site areas, and (4) monitoring speed of construction resources for reducing transportation accidents.

Though to demonstrate a proof-of-concept system application, the experimental setup is implemented in a building environment, but the focus of the study is to understand the movements of construction resources on a site. Deploying the proposed system on a typical construction site will not compromise its utility but will raise concerns in the process of location data acquisition. Placement of beacons and making sure that beacons remain intact to their original positions in the dynamic environment can be challenging. New walls and infrastructure supports are added often on sites, while others are detached, and construction equipment is constantly changing its position that can greatly affect the functionality of beacons and will introduce large measurement errors in the location data. Future work is needed to be done to increase the reliability of the location data by deploying such fault tolerant sensor network that exhibits robustness and having an ability to diagnose the problem quickly in case of any sensor failure. This should be achieved remotely as many locations on a construction site often have limited access and visiting site areas physically and regularly is not possible all the time during construction processes.

Acknowledgements The authors thank the Conseil Régional de Bourgogne-Franche-Comté, the French government for their funding, SATT Grand-Est, and IUT-Dijon (http://iutdijon.u-bourgogne.fr). The authors also want to thank Orval Touitou for his technical assistance to this research work.

Publisher's note Springer Nature remains neutral with regard to jurisdictional claims in published maps and institutional affiliations. 


\section{References}

1. Shao B, Hu Z, Liu Q, Chen S, He W (2018) Fatal accident patterns of building construction activities in China. Saf Sci 111:253-263 (In Press). https://doi.org/10.1016/j.ssci.2018.07.019

2. Poh CQ, Ubeynarayana CU, Goh YM (2018) Safety leading indicators for construction sites: a machine learning approach. Autom Constr 93:375-386. https://doi.org/10.1016/j.autcon.2018.03.022

3. Stats.bls.gov. (2017) Census of fatal occupational injuries (CFOI) current and revised data. http://stats.bls.gov/iif/oshcfoi1.htm. Accessed 28 September 2017

4. Fang W, Ding L, Luo H, Love PE (2018) Falls from heights: a computer vision-based approach for safety harness detection. Autom Constr 91:53-61. https://doi.org/10.1016/j.autcon.2018.02. 018

5. Zhou C, Ding LY (2017) Safety barrier warning system for underground construction sites using internet-of-things technologies. Autom Constr 83:372-389. https://doi.org/10.1016/j.autcon.2017. 07.005

6. Li H, Yang X, Skitmore M, Wang F, Forsythe P (2017) Automated classification of construction site hazard zones by crowd-sourced integrated density maps. Autom Constr 81:328-339. https://doi. org/10.1016/j.autcon.2017.04.007

7. Pradhananga N, Teizer J (2013) Automatic spatio-temporal analysis of construction site equipment operations using GPS data. Autom Constr 29:107-122. https://doi.org/10.1016/j.autcon.2012.09.004

8. Yu Y, Guo H, Ding Q, Li H, Skitmore M (2017) An experimental study of real-time identification of construction workers' unsafe behaviors. Autom Constr 82:193-206. https://doi.org/10.1016/j. autcon.2017.05.002

9. Park MW, Brilakis I (2016) Continuous localization of construction workers via integration of detection and tracking. Autom Constr 72: 129-142. https://doi.org/10.1016/j.autcon.2016.08.039

10. Teizer J, Cheng T (2015) Proximity hazard indicator for workerson-foot near miss interactions with construction equipment and geo-referenced hazard areas. Autom Constr 60:58-73. https://doi. org/10.1016/j.autcon.2015.09.003

11. Awolusi I, Marks E, Hallowell M (2018) Wearable technology for personalized construction safety monitoring and trending: review of applicable devices. Autom Constr 85:96-106. https://doi.org/10. 1016/j.autcon.2017.10.010

12. Cai H, Andoh AR, Su X, Li S (2014) A boundary condition based algorithm for locating construction site objects using RFID and GPS. Adv Eng Inform 28(4):455-468. https://doi.org/10.1016/j. aei.2014.07.002

13. Zheng Y (2015) Trajectory data mining: an overview. ACM Trans Intell Syst Technol 6(3):1-41. https://doi.org/10.1145/2743025

14. Albanna BH, Moawad IF, Moussa SM, Sakr MA (2015) Semantic Trajectories: A Survey from Modeling to Application. In: Semantic trajectories: a survey from modeling to application. Information fusion and geographic information systems. Springer International Publishing, pp 59-76. https://doi.org/10.1007/978-3-319-16667-4

15. Li H, Lu M, Hsu SC, Gray M, Huang T (2015) Proactive behaviorbased safety management for construction safety improvement. Saf Sci 75:107-117. https://doi.org/10.1016/j.ssci.2015.01.013

16. Baslyman M, Rezaee R, Amyot D, Mouttham A, Chreyh R, Geiger G, Stewart A, Sader S (2015) Real-time and location-based hand hygiene monitoring and notification: proof-of-concept system and experimentation. Pers Ubiquit Comput 19:667-688. https://doi.org/ 10.1007/s00779-015-0855-y

17. Lopez-Novoa U, Aguilera U, Emaldi M, López-de-Ipina D, Pérezde-Albeniz I, Valerdi D, Iturricha I, Arza E (2017) Overcrowding detection in indoor events using scalable technologies. Pers Ubiquit
Comput 21(3):507-519. https://doi.org/10.1007/s00779-017-10126

18. Spaccapietra S, Parent C, Damiani ML, de Macedo JA, Porto F, Vangenot C (2008) A conceptual view on trajectories. Data Knowl Eng 65(1):126-146. https://doi.org/10.1016/j.datak.2007.10.008

19. Arslan M, Cruz C, Roxin AM, Ginhac D (2018) Spatio-temporal analysis of trajectories for safer construction sites. Smart and Sustainable Built Environ 7(1):80-100. https://doi.org/10.1108/ SASBE-10-2017-0047

20. Guc B, May M, Saygin Y, Körner C (2008) Semantic annotation of GPS trajectories. Proceedings of the eleventh AGILE international conference on geographic information science. Girona, Spain, pp $1-9$

21. Yan Z, Giatrakos N, Katsikaros V, Pelekis N, Theodoridis Y (2011) SeTraStream: semantic-aware trajectory construction over streaming movement data. Advances in spatial and temporal databases. Lect Notes Comput Sci 6849:367-385. https://doi.org/10.1007/ 978-3-642-22922-0 22

22. Buchin M, Driemel A, Kreveld MV, Sacristan V (2010) An algorithmic framework for segmenting trajectories based on SpatioTemporal Criteria. Proceedings of the 18th SIGSPATIAL International Conference on Advances in Geographic Information Systems New York, NY, USA 202-211. https://doi.org/10.1145/ 1869790.1869821

23. Dabiri S, Heaslip K (2018) Inferring transportation modes from GPS trajectories using a convolutional neural network. Transportation Res. Part C: Emerg Technol 86:360-371. https:// doi.org/10.1016/j.trc.2017.11.021

24. Balzano W, Sorbo MRD (2014) SeTra: a smart framework for GPS trajectories' segmentation. International Conference on Intelligent Networking and Collaborative Systems Salerno, Italy 362-368. https://doi.org/10.1109/INCoS.2014.106

25. Sankararaman S, Agarwal PK, Mølhave T, Pan J, Boedihardjo AP (2013) Model-driven matching and segmentation of trajectories. Proceedings of the 21st ACM SIGSPATIAL International Conference on Advances in Geographic Information Systems New York, NY, USA, pp 234-243. https://doi.org/10.1145/ 2525314.2525360

26. Panagiotakis C, Pelekis N, Kopanakis I, Ramasso E, Theodoridis Y (2012) Segmentation and sampling of moving object trajectories based on representativeness. IEEE Trans Knowl Data Eng 24(7): 1328-1343. https://doi.org/10.1109/TKDE.2011.39

27. Yan Z, Chakraborty D, Parent C, Spaccapietra S, Aberer K (2011) SeMiTri: a framework for semantic annotation of heterogeneous trajectories. Proceedings of the 14th International Conference on Extending Database Technology (EDBT/ICDT '11), ACM, New York, NY, USA :259-270. https://doi.org/10.1145/1951365. 1951398

28. Yan Z (2011) Semantic trajectories: computing and understanding mobility data. Doctoral dissertation. Lausanne, EPFL. https://doi. org/10.5075/epfl-thesis-5144

29. Wu F, Li Z, Lee WC, Wang H, Huang Z (2015) Semantic annotation of mobility data using social media. Proceedings of the 24th International Conference on World Wide Web. Florence, Italy, pp 1253-1263. https://doi.org/10.1145/2736277.2741675

30. Furletti B, Cintia P, Renso C, Spinsanti L (2013) Inferring human activities from GPS tracks. Proceedings of the 2nd ACM SIGKDD International Workshop on Urban Computing (UrbComp '13), Chicago, Illinois, USA;1-8. https://doi.org/10.1145/2505821. 2505830

31. Nogueira TP, Braga RB, de Oliveira CT, Martin H (2018) FrameSTEP: a framework for annotating semantic trajectories based on episodes. J of Expert Systems with Appl 92:533-545. https://doi.org/10.1016/j.eswa.2017.10.004

32. de Graaff V, de By RA, van Keulen M (2016) Automated semantic trajectory annotation with indoor point-of-interest visits in urban 
areas. Proceedings of the 31st Annual ACM Symposium on Applied Computing (SAC '16), Pisa, Italy: 552-559. https://doi. org/10.1145/2851613.2851709

33. Fileto R, May C, Renso C, Pelekis N, Klein D, Theodoridis Y (2015) The Baquara2 knowledge-based framework for semantic enrichment and analysis of movement data. Data Knowl Eng 98: 104-122. https://doi.org/10.1016/j.datak.2015.07.010

34. Wu F, Wang H, Li Z, Lee WC, Huang Z (2015) SemMobi: a semantic annotation system for mobility data. Proceedings of the 24th International Conference on World Wide Web (WWW '15 Companion), Florence, Italy: 255-258. https://doi.org/10.1145/ 2740908.2742837

35. Wan C, Zhu Y, Yu J, Shen Y (2018) SMOPAT: mining semantic mobility patterns from trajectories of private vehicles. Inf Sci 429: 12-25. https://doi.org/10.1016/j.ins.2017.10.043

36. Cai G, Lee K, Lee I (2018) Mining mobility patterns from geotagged photos through semantic trajectory clustering. Cybern Syst 49(4):234-256. https://doi.org/10.1080/01969722.2018. 1448236

37. Heijden K (2005) Scenarios: the art of strategic conversation, 2nd edn. John Wiley \& Sons, Hoboken

38. Carroll JM (2009) Scenario based design. Handbook of humancomputer interaction, 2nd edn. North-Holland, pp 383-406. https://doi.org/10.1016/B978-044481862-1.50083-2

39. Kontakt (2018) "Beacons," available at:https://kontakt.io/blebeacons-tags/. Last Accessed 05 Dec 2018

40. Lu CT, Lei PR, Peng WC, Su J (2011) A framework of mining semantic regions from trajectories. In: International Conference on Database Systems for Advanced Applications. Springer, Berlin, Heidelberg, pp 193-207. https://doi.org/10.1007/978-3-64220149-3 16

41. Ashbrook D, Starner T (2003) Using GPS to learn significant locations and predict movement across multiple users. Pers Ubiquit Comput 7(5):275-286. https://doi.org/10.1007/s00779-003-0240-0

42. Palma AT, Bogorny V, Kuijpers B, Alvares LO (2008) A clusteringbased approach for discovering interesting places in trajectories. Proceedings of the ACM symposium on Applied computing, New York, NY, USA: 863-868. https://doi.org/10.1145/1363686. 1363886

43. Cruz C (2017) Semantic trajectory modeling for dynamic built environments, IEEE International Conference on Data Science and Advanced Analytics (DSAA), pp 468-476

44. Fileto R, Krüger M, Pelekis N, Theodoridis Y, Renso C (2013) Baquara: a holistic ontological framework for movement analysis using linked data. International Conference on Conceptual Modeling, Springer, Berlin, Heidelberg, pp 342-355

45. Bogorny V, Renso C, de Aquino AR, de Lucca Siqueira F, Alvares LO (2014) Constant-a conceptual data model for semantic trajectories of moving objects. Trans GIS 18(1):66-88

46. Mohammadi MS, Isabelle M, Thérèse L, Christophe F (2017) A semantic modeling of moving objects data to detect the remarkable behavior. AGILE, Wageningen

47. Yan Z, Parent C, Spaccapietra S, Chakraborty D (2010) A hybrid model and computing platform for spatio-semantic trajectories. Extended Semantic Web Conference: 60-75, Springer, Berlin, Heidelberg. https://doi.org/10.1007/978-3-642-13486-9 5

48. Zhang S, Teizer J, Pradhanang N (2015) Global positioning system data to model and visualize workspace density in construction safety planning. Proceedings of the 32nd International Symposium on Automation and Robotics in Construction, Oulu, Finland, pp 1-7. https://doi.org/10.22260/ISARC2015/0041

49. Costin AM, Teizer J, Schoner B (2015) RFID and BIM-enabled worker location tracking to support real-time building protocol and data visualization. Journal of Information Technology in Construction (ITcon) 20(29):495-517 http://www.itcon.org/2015/ 29

50. Mahmoud H, Akkari N (2016) Shortest path calculation: a comparative study for location-based recommender system. World Symposium on Computer Applications \& Research (WSCAR), Cairo, pp 1-5. https://doi.org/10.1109/WSCAR.2016.16

51. Heng L, Shuang D, Skitmore M, Qinghua H, Qin Y (2016) Intrusion warning and assessment method for site safety enhancement. Saf Sci 84:97-107. https://doi.org/10.1016/j.ssci.2015.12. 004

52. Carbonari A, Giretti A, Naticchia B (2011) A proactive system for real-time safety management in construction sites. Autom Constr 20(6):686-698. https://doi.org/10.1016/j.autcon.2011.04.019

53. Arslan M, Cruz C, Ginhac D (2018) Semantic enrichment of spatiotemporal trajectories for worker safety on construction sites. Procedia Computer Science 130:271-278. https://doi.org/10.1016/ j.procs.2018.04.039

54. Riaz Z, Arslan M, Kiani AK, Azhar S (2014) CoSMoS: a BIM and wireless sensor based integrated solution for worker safety in confined spaces. Autom Constr 45:96-106. https://doi.org/10.1016/j. autcon.2014.05.010

55. Autodesk (2018) [online] Available at: http://paulaubin.com/ downloads/2017_AU/BIM128338-Aubin-AU2017.pdf. Accessed 13 Sep 2018 